\title{
Evidence for Winding States in Noncommutative Quantum Field Theory
}

\author{
W. Fischler, E. Gorbatov, A. Kashani-Poor, S. Paban, P. Pouliot \\ Department of Physics \\ University of Texas, Austin, TX 78712 \\ E-mail: fischler, elie, kashani, \\ paban, pouliot@physics.utexas.edu \\ Joaquim Gomis \\ Departament ECM, Facultat de Fisica \\ Universitat de Barcelona \\ and \\ Institut de Fisica d'Altes Energies \\ Diagonal 647, E-08028 Barcelona, Spain \\ E-mail:gomis@ecm.ub.es
}

\begin{abstract}
We study noncommutative field theories at finite temperature to learn more about the degrees of freedom in the non-planar sector of these systems. We find evidence for winding states. At temperatures for which the thermal wavelength is smaller than the noncommutativity scale, there is a drastic reduction of the degrees of freedom in the non-planar sector. In this regime, the non-planar sector has thermodynamics resembling that of a $1+1$ dimensional field theory.
\end{abstract}

KEYWORDS: noncommutative geometry, quantum field theory, supersymmetry, string theory, thermodynamics, winding states. 


\section{Contents}

1. Introduction $\quad 1$

2. The thermodynamics of noncommutative $g^{2} \phi^{4}$ in perturbation theory 2

3. The thermodynamics of the noncommutative Wess-Zumino model 5

4. The thermodynamics of noncommutative $g^{2} \phi^{4}$ in the "mean field" $\begin{array}{ll}\text { approximation } & 8\end{array}$

5. Winding states around spatial cycles $\quad 10$

$\begin{array}{ll}\text { 6. Conclusions } & 11\end{array}$

A. UV divergences in $g^{2} \phi^{4} \quad 12$

B. IR divergences in $g^{2} \phi^{4} \quad 13$

\section{Introduction}

Gauge theories on noncommutative spaces can be obtained by taking the infinite tension limit of string theory in the presence of a very strong B-field $[1,3,4,2]$. One can thus look upon these field theories as interesting limits of string theory in such an extreme environment. Regardless of their ancestry, these field theories have very interesting properties [5]-[28], making their study fascinating in its own right. Many unusual properties already emerge in scalar field theories. In particular, one sees the emergence of unexpected infrared behavior in correlation functions [4] (we will refer to this paper in the following as MSV) unlike anything seen in conventional field theories. These long distance correlations appear in massive theories and are not related to any massless fields in the Lagrangian. Rather, these infrared effects are due to the Moyal phases. As can be seen in Feynman diagrams, it is the integration over the high ultraviolet modes that is responsible for this peculiar infrared behavior. The UV and IR are intertwined in a manner never seen in local field theory on commutative spaces. MSV have a 
proposal for what kind of degrees of freedom are responsible for these IR correlations: their claim is that in the zero slope limit and large B field, the closed string states do not decouple.

To gain some more insight into the structure of these noncommutative field theories, we subject them to a heat bath and consider various thermodynamic quantities. We will show that the partition functions of these theories get contributions at high temperature from configurations winding around the temporal direction. This implies that there are extended objects in these theories that have the ability to wind, which seems consistent with the suggestions of MSV. We also test this picture by compactifying the spatial commuting direction to a circle and again find contributions to the partition function, this time from configurations winding around the spatial circle.

It is useful to consider a simple field theory like massive $\phi^{4}$ and the supersymmetric Wess-Zumino model. We were motivated to include a supersymmetric example in order to show that the existence of winding states persists in theories where the sensitivity to the UV physics is softer. Because of the special UV properties of the supersymmetric case, the IR sensitivity is milder and allows us to limit ourselves to the lowest order in perturbation theory when calculating thermodynamic quantities.

The paper is organized as follows: in section 2 we will describe in some detail the thermodynamics of noncommutative $\phi^{4}$. There we present the calculation of the free energy to $O\left(g^{2}\right)$ and discuss the various divergences and also show how the winding states appear. In section 3, we investigate whether supersymmetric systems whose UV behavior are less singular affect the existence of winding states. For this purpose, we consider the Wess-Zumino model and find again the existence of winding states. We calculate the free energy, from which the entropy, internal energy and specific heat can be derived. In section 4 , we return to the $\phi^{4}$ theory and show how to calculate the free

energy in the "mean field" approximation. In section 5, we discuss the implications of these results and briefly repeat the calculations at zero and finite temperature for the case where the sole commuting direction is a circle. We show that at finite temperature, there are no membrane states wrapping around the torus spanned by the temporal and $x_{3}$ cycles. Instead, we find winding states winding each of the cycles independently.

We then conclude with some speculations.

\section{The thermodynamics of noncommutative $g^{2} \phi^{4}$ in perturba- tion theory}

We present a perturbative calculation of the free energy, F, which assumes that the coupling constant $g^{2}$ is small. As we will see, the appearance of IR divergences invalidates 
the perturbative expansion. Nevertheless, we believe it is instructive to go through this exercise as it already shows the existence of winding states. Later in section 4 we will have to use a mean field approximation to ensure that long range correlations are properly screened.

In this paper, we will restrict ourselves to the case where the noncommutativity is solely among the spatial directions.. Without loss of generality then in this 4-dimensional case, we can choose the $(1,2)$ plane to be noncommutative and leave time and the third spatial dimension commutative.

At finite temperature, the Feynman rules are the ones that correspond to a theory with "time" being a circle of radius $\beta$, where $\beta$ is the inverse temperature, $\beta=1 / T$. This implies that the integrals over frequencies in diagrams are replaced by discrete sums over the so-called Matsubara frequencies. The noncommutativity in the $(1,2)$ plane manifests itself in Feynman diagrams through the appearance of Moyal phases in vertices. These Moyal phases depend on momenta in the $(1,2)$ directions.

When evaluating the contributions to the free energy, one needs to consider both planar and non-planar diagrams. The planar contributions are very much like in the commutative theory except for some combinatoric factors [5]. Inspite of the changed combinatorics, the behavior in the UV is still controlled by zero temperature physics. In order to illustrate the divergences that occur in the perturbative expansion of the free energy in the noncommutative theory, we will first concentrate on the non-planar contributions to lowest order in $g^{2}$. This will also enable us to show the appearance of contributions from winding states to the free energy.

The leading non-planar contribution at order $g^{2}$ to the free energy comes from a two-loop diagram.
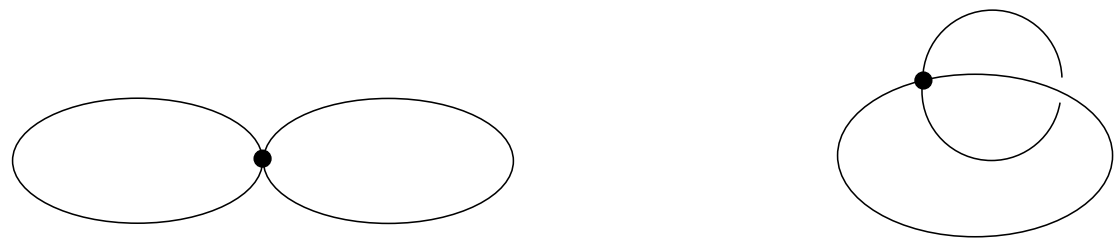

Fig.1: Planar and nonplanar two loop contributions to the free energy.

This diagram is divergent, where some of the divergences can be interpreted as originating in the UV and some divergence can be attributed to the IR. The UV divergences will be shown to arise from zero temperature physics and similar UV divergences will appear in higher order terms in the expansion in $g^{2}$. Also, in a manner similar to the IR divergences that appears at lowest order, each successive order of perturbation 
theory will be plagued by IR divergences. These IR divergences grow more singular with increasing order in the perturbative expansion, invalidating the expansion. We will return to the formal application of this mean field approximation in section 4 after the study of the $O\left(g^{2}\right)$ contribution to the free energy in the Wess-Zumino model.

The contribution to the free energy from this two-loop non-planar diagram is:

$$
-g^{2} T^{2} \sum_{n=-\infty}^{\infty} \sum_{l=-\infty}^{\infty} \int \frac{d^{3} p}{(2 \pi)^{3}} \frac{d^{3} k}{(2 \pi)^{3}} \frac{e^{i p \theta k}}{\left(\frac{4 \pi^{2} n^{2}}{\beta^{2}}+p^{2}+M^{2}\right)\left(\frac{4 \pi^{2} l^{2}}{\beta^{2}}+k^{2}+M^{2}\right)}
$$

where $p \theta k=\theta\left(p_{1} k_{2}-p_{2} k_{1}\right)$ and $p^{2}=p_{1}{ }^{2}+p_{2}{ }^{2}+p_{3}{ }^{2}$.

We will be interested in temperatures much larger than the mass, so that to leading order in $\beta M$, it can be neglected.

The integration over the momenta can be done by introducing Schwinger parameters. In order to show how contributions of winding configurations appear, we will look at equation (2.1) in some detail. The integral appearing in this expression can after some manipulations be rewritten as:

$$
-g^{2} T \sum_{n, l} \int \frac{d^{3} p}{(2 \pi)^{3}} \frac{1}{\left(\frac{4 \pi^{2} n^{2}}{\beta^{2}}+p^{2}\right)\left(4 \pi^{2}\left(l^{2} \beta^{2}+|\theta p|^{2}\right)+\Lambda^{-2}\right)},
$$

where $|\theta p|=\theta\left(p_{1}^{2}+p_{2}^{2}\right)^{1 / 2}$ and $\Lambda$ is an ultraviolet cutoff similar to the one used by MSV. We also have neglected the mass in obtaining this expression since we are working in the regime $\beta M \ll 1$.

The sums over $n$ and $l$ can be performed and will lead to the following formula involving Bose distributions

$$
-g^{2} T \int \frac{d^{3} p}{(2 \pi)^{3}} \frac{1+2 n_{\beta}(|p|)}{2|p|} \frac{1+2 n_{1 / \beta}(2 \pi|\theta p|)}{4 \pi|\theta p|},
$$

where $|p|=\left(p_{1}{ }^{2}+p_{2}{ }^{2}+p_{3}{ }^{2}\right)^{1 / 2}$ and $n_{\beta}(|p|)=\frac{1}{e^{\beta|p|}-1}$ is a Bose distribution at temperature $T=1 / \beta$.

Note the appearance in the last expression of two Bose distributions, one at temperature $T$ and the other at temperature $1 / T$. This would be a rather bizarre state of affair for a system in thermal equilibrium. One possibility is that the system is not in equilibrium, but why then in this very special way, with one set of the degrees of freedom distributed thermally at temperature $T$ and the other at temperature $1 / T$ ? We will choose the option that the system is indeed in equilibrium, and that there are configurations, if one thinks in a path integral approach to the partition function, that 
wind around the temporal circle. If one looks at the integral appearing in equation (2.3), one can see the contributions of winding states with momentum $|\theta p|$ to the free energy.

Another interesting feature of equation (2.3) is that the $p_{3}=0$ sector is invariant under the interchange of $\beta$ and $2 \pi \theta / \beta$. This transformation looks like T-duality where the string scale $l_{s}^{2}$ is replaced by $\theta$. We should remind the reader though that this property was obtained in the $\beta M \ll 1$ limit. We do not know whether this duality invariance persists beyond this order to the full theory.

The expression in equation (2.3) has UV divergences and IR divergences. The

IR divergence is linear and comes from the small $\left(p_{1}, p_{2}\right)$ region of integration. This divergence, as will be shown later, is cured by the mean field approximation.

The UV divergent terms in the non-planar sector are calculated in the appendix. Apart from a zero temperature contribution to the vacuum energy, there is a linearly divergent term which is also linear in the temperature. This is a rather unusual contribution to the free energy. Indeed, this term does not contribute to the internal energy of the system but does provide a temperature independent contribution to the entropy. This contribution to the entropy is due to the degeneracy of the winding states. As can be seen in the Bose distribution, their dispersion relation does not involve the momentum along the commuting spatial dimension, $p_{3}$. The very large number of winding states with momenta $p_{1,2}=0$ are the source of the linear divergence. This divergence disappears when screening of the large distance correlations is taken into account. The supersymmetric case does not have such a divergence at the leading order in $g^{2}$ because of its milder behavior in the IR.

\section{The thermodynamics of the noncommutative Wess-Zumino model}

The Lagrangian for the noncommutative Wess-Zumino model is:

$$
\mathcal{L}=i \partial_{\mu} \bar{\psi} \bar{\sigma}^{\mu} \psi+A^{*} \square A-\frac{1}{2} M \psi \psi-\frac{1}{2} M \bar{\psi} \bar{\psi}-g \psi * \psi A-g \bar{\psi} * \bar{\psi} A^{*}-F^{*} F
$$

where $F$ is given by

$$
F=-M A^{*}-g A^{*} * A^{*} .
$$

The calculation of the free energy to $O\left(g^{2}\right)$ proceeds along the same lines as in the bosonic case. In this case there are additional contributions due to new interactions among the bosons and bosons with fermions. The fermions, as usual, have frequencies that are odd multiples of the temperature. 
Three pairs of diagrams contribute at order $g^{2}$, each pair consisting of a planar and a non-planar piece. They sum to give

$$
\begin{aligned}
F / V & =-g^{2} \int \frac{d^{3} p}{(2 \pi)^{3}} \frac{d^{3} k}{(2 \pi)^{3}} \frac{1+e^{i p \theta k}}{\omega_{p} \omega_{k}}\left(n_{B}\left(\omega_{p}\right)+n_{F}\left(\omega_{p}\right)\right)\left(n_{B}\left(\omega_{k}\right)+n_{F}\left(\omega_{k}\right)\right) \\
& +g^{2} T^{4} \mathcal{O}\left(\frac{M^{2}}{T^{2}}\right)
\end{aligned}
$$

with $\omega_{p}=\sqrt{p^{2}+M^{2}}$ and $n_{B, F}\left(\omega_{p}\right)=1 /\left(e^{\beta \omega_{p}} \mp 1\right)$. Notice that this expression for the free energy at high temperature $(\beta M \ll 1)$ is "T- duality invariant" as in the bosonic case. This property applies to the sector with zero momentum along the commuting direction. This result can be seen by expanding one of the Bose distributions in a series of exponentials,

$$
\frac{1}{e^{\beta\left(p^{2}+M^{2}\right)^{1 / 2}}-1}=\sum_{n=1}^{\infty} e^{-\beta n\left(p^{2}+M^{2}\right)^{1 / 2}} .
$$

Then performing the integral over $d^{3} p$, neglecting the mass, produces the series,

$$
\sum_{n=1}^{\infty} \frac{1}{n^{2} \beta^{2}+|\theta k|^{2}}
$$

A similar expression can be obtained for the integration over the Fermi distributions, except that in the fermion case the series is an alternating one. The presence of winding states is then clear as well as the "T- duality invariance" of the free energy.
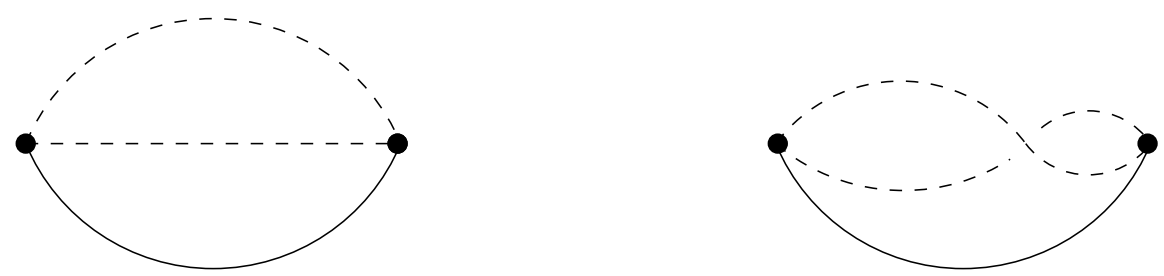

Fig.2: Planar and non-planar two loop fermionic contributions to the free energy.
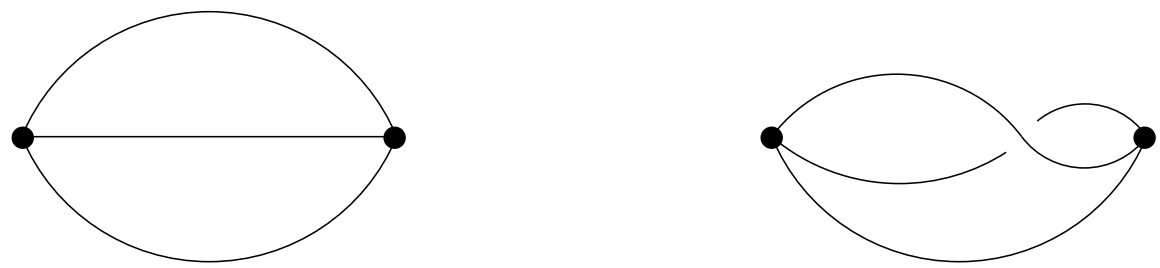

Fig.3: Planar and non-planar two loop bosonic contributions to the free energy. 
The expression (3.1) for the non-planar contribution to the free energy can be further evaluated to yield

$$
-\frac{(2 \pi)^{3}}{\sqrt{2}} \frac{g^{2}}{\theta \beta^{2}} \int_{0}^{\pi} d \eta \int_{0}^{\infty} d q \frac{\tanh \left(\frac{\pi \sin (\eta)}{2} \frac{\theta q}{\beta^{2}}\right)}{\sinh q} .
$$

At high temperature, $\beta M \ll 1$, we consider two limiting cases, $T^{2} \ll 1 / \theta$ and $T^{2} \gg 1 / \theta .^{1}$ The first limit corresponds to the case where the thermal wavelength is bigger than the length scale associated to the noncommutativity. In this limit, one might expect to recover conventional high temperature behavior. Indeed, the leading non-planar contribution to the free energy here is

$$
\left.\frac{F}{V}\right|_{\mathrm{np}} \sim-g^{2} T^{4}
$$

In the other limit, $T^{2} \gg 1 / \theta$, the thermal wavelength is smaller than the noncommutativity scale. We therefore expect novel behavior. We find the free energy in this regime to be

$$
\left.\frac{F}{V}\right|_{\mathrm{np}} \sim-g^{2} \frac{T^{2}}{\theta} \log T^{2} \theta
$$

where the logarithm was obtained by numerical methods. In this case, the contribution to the free energy resembles the contribution of a massless gas in $1+1$ dimension, modified by a logarithm.

One is tempted to conclude from these results that there is a drastic reduction of degrees of freedom in the non-planar sector! For thermal wavelengths larger than the noncommutativity scale, the system behaves as a relativitic $3+1$ dimensional gas. For wavelengths smaller than this scale, the system behaves roughly like a $1+1$ dimensional gas. As the temperature is raised past $1 / \theta^{1 / 2}$ it looks like the winding states do behave as strings. Up to the logarithm, one is reminded by the temperature dependence in equation (3.4) of the result obtained by Atick and Witten [29] for the high temperature behavior of strings. We do not know whether this is just a coincidence or whether there is some deeper significance to this behavior. A test of this high temperature dependence of the free energy due to the non planar sector would be to study higher dimensional examples where there are more noncommuting spatial dimensions. It is amusing to note that the non-planar sector of this system exhibits, to leading order at very high temperature, the equation of state $p=\rho, p$ being pressure and $\rho$ energy density.

\footnotetext{
${ }^{1}$ We also assume that $\theta M^{2} \ll 1$, i.e. the Compton wavelength of the scalars is larger than the length scale associated to the noncommutativity.
} 


\section{The thermodynamics of noncommutative $g^{2} \phi^{4}$ in the "mean field" approximation}

We now go back to the noncommutative $g^{2} \phi^{4}$ theory of section 2 . There, the appearance of IR divergences ruins the validity of the perturbative expansion (in $g^{2}$ ) for the free energy. This is a familiar phenomenon in thermal physics. For example, in the case of a plasma of electric charges in QED, one finds IR divergences in perturbation theory for the free energy. This can be traced to the large distance behavior of the correlation function, $\langle\rho(x) \rho(0)\rangle$. This IR divergence disappears after summing a series of diagrams contributing to the correlation function between charge densities, $\langle\rho(x) \rho(0)\rangle$. This series of diagrams is a geometric series in the one-particle irreducible charge density correlation function. When this series is summed, one finds that the charge densities are screened. The screening mass or Debye mass is the IPI charge density correlation function evaluated at zero momentum.

In the case of noncommutative $\phi^{4}$, the IR divergence already appears at zero temperature and is again screened by summing a geometric series in the one-particle irreducible two-point function (MSV).

In the finite temperature case, we will approach the problem of IR divergences by treating the gas in a mean field approximation. In this approximation, each quantum of the scalar field is moving in the average field produced by the other scalars. Unlike more traditional cases where the mean field approximation is used, this case has the particular feature that the mean field will depend on the momentum of the particle that moves in this background. This momentum lies in the noncommutative plane. Using this approximation, we will show how the long range correlations are screened.

The Hamiltonian $\mathrm{H}$ is:

$$
H=\frac{1}{2} \int d^{3} x\left(\Pi^{2}(x)+(\nabla \phi(x))^{2}+M^{2} \phi^{2}(x)\right)+g^{2} \int d^{3} x \phi(x) * \phi(x) * \phi(x) * \phi(x) .
$$

The free energy $\mathrm{F}$ is obtained from the logarithm of the partition function $Z$, where $Z=\operatorname{tr} e^{-\beta H}$, as $-\beta F=\log Z$. When $g^{2}$ is small, it can be formally written as $F_{0}$, the free gas contribution to $\mathrm{F}$, corrected by $O\left(g^{2}\right)$ contributions. We should remind the reader that this procedure fails because of IR divergences. We present this expression for $\mathrm{F}$ here because it will be useful when we get to the mean field approximation:

$$
\frac{\beta F}{V}=\int \frac{d^{3} p}{(2 \pi)^{3}}\left[\frac{\beta}{2}\left(p^{2}+M^{2}\right)^{1 / 2}+\log \left(1-\exp \left(-\beta\left(p^{2}+M^{2}\right)^{1 / 2}\right]+O\left(g^{2}\right) .\right.\right.
$$

The contribution from the free gas to the previous formula could have been obtained by calculating a one-loop diagram in the path integral formulation. 
In the mean field approximation, a picture emerges where the system looks like a free gas for which the dispersion formula for the energy of a quantum is modified by the average effect of the other quanta.

Formally, this will be implemented in the path integral by using the following action:

$$
S=\int_{0}^{\beta} d \tau \int \frac{d^{3} k}{(2 \pi)^{3}}\left[\left(\partial_{t} \phi\right)^{2}(k)-k^{2} \phi^{2}(k)-M^{2} \phi^{2}(k)-g^{2}\left\langle\phi^{2}\right\rangle_{\theta k} \phi^{2}(k)\right] .
$$

where $\left\langle\phi^{2}\right\rangle_{\theta k}$ satisfies an integral equation:

$$
\left\langle\phi^{2}\right\rangle_{\theta k}=1 / \beta \sum_{n} \int \frac{d^{3} p}{(2 \pi)^{3}} \frac{2+e^{i p \theta k}}{\frac{4 \pi^{2} n^{2}}{\beta^{2}}+p^{2}+M^{2}+g^{2}\left\langle\phi^{2}\right\rangle_{\theta p}} .
$$

We will not solve this integral equation exactly but rather find the solution to it as a power series expansion in $g^{2}$. In this paper we will confine ourselves to work with the solution to leading order.

In the limit of high temperature, $M \beta \ll 1$, we neglect to first order the mass $\mathrm{M}$ and we sum over $n$, and obtain to leading order $\left\langle\phi^{2}\right\rangle_{\theta k}$.

$$
\begin{aligned}
\left\langle\phi^{2}\right\rangle_{\theta k} & =\sum_{n} \frac{1}{4 \pi^{2} n^{2} \beta^{2}+(2 \pi|\theta k|)^{2}+\Lambda^{-2}} \\
& =\frac{1}{2 \beta \sqrt{(2 \pi|\theta k|)^{2}+\Lambda^{-2}}}\left(1+2 n_{1 / \beta}\left(\sqrt{4 \pi^{2}|\theta k|^{2}+\Lambda^{-2}}\right) .\right.
\end{aligned}
$$

Notice that the Bose distribution in the expression for $\left\langle\phi^{2}\right\rangle$ is unusual as it distributes $k_{1,2}$ according to a "temperature" $\tau$, where $\tau=1 / \theta T$, i.e. proportional to the inverse temperature. One can interpret this property, as was argued earlier, to be evidence for winding states. Indeed, the summation that appears in $\left\langle\phi^{2}\right\rangle$ is over an integer multiple of the length of the temporal circle rather than the inverse radius as would be the case for momenta. The free energy can then be written as a "tree" contribution plus a one-loop contribution.

$$
\begin{gathered}
\frac{\beta F}{V}=M^{2}\left\langle\int d^{4} x \phi^{2}(x)\right\rangle+\frac{\beta g^{2}}{V}\left\langle\int d^{4} x \phi(x) * \phi(x) * \phi(x) * \phi(x)\right\rangle+ \\
\int \frac{d^{3} p}{(2 \pi)^{3}}\left[\frac{\beta}{2} \sqrt{p^{2}+M^{2}+g^{2}\left\langle\phi^{2}\right\rangle_{\theta p}}+\log \left(1-\exp \left(-\beta\left(p^{2}+M^{2}+g^{2}\left\langle\phi^{2}\right\rangle_{\theta p}\right)^{1 / 2}\right)\right] .\right.
\end{gathered}
$$

In this last equation, the average value of

$$
M^{2} \int d^{4} x \phi^{2}(x)+\frac{\beta g^{2}}{V} \int d^{4} x \phi(x) * \phi(x) * \phi(x) * \phi(x)
$$


is evaluated by using the action S, equation (4.1), in a path integral calculation.

As expected and demonstrated explicitly in the appendix, the IR divergences disappear.

The one-loop contribution

$$
\int \frac{d^{3} p}{(2 \pi)^{3}}\left[\frac{\beta}{2} \sqrt{p^{2}+M^{2}+g^{2}\left\langle\phi^{2}\right\rangle_{\theta p}}+\log \left(1-\exp \left(-\beta\left(p^{2}+M^{2}+g^{2}\left\langle\phi^{2}\right\rangle_{\theta p}\right)^{1 / 2}\right)\right]\right.
$$

can be visualized as a single quantum of the scalar field propagating around a loop with a momentum that at $O\left(g^{0}\right)$ is thermally distributed at temperature $T$. This particle is moving in the presence of a background, $\left\langle\phi^{2}\right\rangle$, which it interacts with at $O\left(g^{2}\right)$ and that consists of states winding around the temporal circle of radius $1 / T$. These winding states have momenta solely in the noncommutative plane. It is quite tempting to think of them as closed strings reminiscent of the suggestions made in MSV.

To be complete, one should study the UV divergences and see whether they are associated to zero temperature effects. We will not attempt this study here. It should be kept in mind that it is not completely clear yet whether this theory is renormalizable, even though some evidence exists.

\section{Winding states around spatial cycles}

The presence of winding states, which in the finite temperature case wind around the temporal circle, can also be noticed when one compactifies the commuting spatial dimension, $x_{3}$. The states winding around $x_{3}$ can be detected for example if we calculate the free energy for this case.

The calculation of the previous section can be repeated, with each $\int \frac{d p_{3}}{2 \pi}$ replaced by $\frac{1}{2 \pi L} \sum_{l}$, where 1 is an integer.

To show the existence of winding states, it is sufficient to again consider the integral equation for the two point function:

$$
\left\langle\phi^{2}\right\rangle_{\theta k}=\frac{1}{\beta L} \sum_{n, l} \int \frac{d^{2} p}{(2 \pi)^{2}} \frac{e^{i p \theta k}}{\frac{4 \pi^{2} n^{2}}{\beta^{2}}+\frac{4 \pi^{2} l^{2}}{L^{2}}+p^{2}+M^{2}+g^{2}\left\langle\phi^{2}\right\rangle_{\theta p}} .
$$

In the limit of high temperature, $\beta M \ll 1$, and small radius, $L M \ll 1$, we find for $\left\langle\phi^{2}\right\rangle_{\theta k}$, upon performing the $\int d^{2} p$,

$$
\left\langle\phi^{2}\right\rangle_{\theta k}=\sum_{n, l} \frac{1}{4 \pi^{2} n^{2} \beta^{2}+4 \pi^{2} l^{2} L^{2}+(2 \pi|\theta k|)^{2}+\Lambda^{-2}}
$$


This last expression clearly shows the presence of winding states around both circles. Again these states become prevalent in the small radii limit, which is to be expected since in these limits these states are very "light".

This formula for $\left\langle\phi^{2}\right\rangle_{\theta k}$ also suggests that there are no wrapping states around the 2-torus spanning the temporal and $x_{3}$ dimensions, which would look like an additional piece $(n l \beta L)^{2}$ in the denominator of this equation.

The same result holds in the supersymmetric case. In some ways, this could have been expected. Imagine subjecting this system to a thermal environment. In this case, the bosons and fermions have different distributions and we should not therefore expect a drastically different behavior from the non-supersymmetric cases.

One might speculate about the existence of multidimensional extended objects in higher dimensional theories where several $\theta_{i j} \neq 0$. To test this, we briefly consider the example of the six-dimensional $\phi^{3}$ theory, with various $\theta_{i j} \neq 0$.

At high temperature and after compactifying the commuting $x_{5}$ on a small circle, it should be possible to see whether or not wrapping states emerge. This can be tested, as we learned above, simply by calculating $\left\langle\phi^{2}\right\rangle_{\theta p, \theta^{\prime} p^{\prime}}$. We obtain

$$
\left\langle\phi^{2}\right\rangle_{\theta p, \theta^{\prime} p^{\prime}}=\sum_{l, n} \frac{1}{\left(-4 \pi^{2} p_{i} \theta^{i j} \theta_{j k} p^{k}+4 \pi^{2} \beta^{2} n^{2}+4 \pi^{2} L^{2} l^{2}\right)^{2}} .
$$

We thus conclude that there are no wrapping states, just winding states.

\section{Conclusions}

What is the physics that is responsible for the existence of these winding states?

Clearly the UV-IR connection that exists in the noncommutative plane is crucial. The expressions which reveal the existence of the winding states show that this peculiar IR behavior originates in the UV region of integration. One might then conclude that the uncertainty relationship between $x_{1}$ and $x_{2}$ in the case where $\theta_{12} \neq 0$ is implemented by or implies the existence of one-dimensional structures. In other words, measuring $x_{1}$ sharply renders the other direction $x_{2}$ totally uncertain and maybe this is intimately related to the existence of extended one-dimensional objects in a rather mysterious way. These "strings" can then wind around various cycles. However, we did not find multidimensional objects wrapping multicycles in higher dimension.

We have discovered from studying the high temperature regime of the supersymmetric theory the existence of two different behaviors. We believe that these results are valid in general, but we have only been able to calculate reliably to leading order in the supersymmetric case. When the thermal wavelength is bigger than the noncommutativity length scale, we find that the non-planar sector behaves as a conventional $3+1$ 
dimensional relativistic gas. For the thermal wavelength smaller than the noncommutativity scale, we observe a reduction of the degrees of freedom in the non-planar sector. The non-planar sector now behaves as a $1+1$ (not, as might have been expected, $2+1$ ) dimensional gas. This seems to indicate a drastic reduction of the degrees of freedom in that sector. Equivalently, we find the equation of state of this sector is approximately that of an incompressible gas with equation of state $p=\rho$.

It would be interesting to see whether this behavior persists in higher dimensional theories.

In the theories we have studied, the fascinating behavior of the non-planar sector is overshadowed by the planar sector. Thus, the challenging question remains of what theory captures exclusively the physics of the non-planar sector.

\section{Acknowledgments}

The work of WF, EG, JG, AK-P, SP, PP is supported in part by the Robert Welch Foundation and the NSF under grant number PHY-9511632, JG is partially supported by AEN 98-0431 (CICYT), GC 1998SGR (CIRIT), SP is also supported by NSF grant PHY-9973543.

\section{A. UV divergences in $g^{2} \phi^{4}$}

Of the four terms in equation (2.3), the two damped by the Bose distribution $n_{\beta}(|p|)$ are obviously UV convergent. The remaining two give rise to a linear and a logarithmic UV

divergence (we remind the reader that the argument $|\theta p|$ of the second Bose distribution $n_{1 / \beta}$ appearing in equation (2.3) is independent of $p_{3}$ ).

The term

$$
-g^{2} T \int \frac{d^{3} p}{(2 \pi)^{3}} \frac{n_{1 / \beta}(|\theta p|)}{|p| 4 \pi|\theta p|}
$$

is divergent in the region $p_{3} \gg \sqrt{p_{1}^{2}+p_{2}^{2}}$. In this region, we obtain

$$
-\frac{1}{2(2 \pi)^{3}} \frac{g^{2} T}{\theta} \log \Lambda \int d p_{1,2} n_{1 / \beta}(|\theta p|)=-\frac{1}{2(2 \pi)^{3}} \frac{g^{2}}{\theta^{2}} \log \Lambda \int d x \frac{1}{e^{2 \pi x}-1} .
$$

Notice that the $x$ integral is IR divergent. If we introduce an IR cutoff to regulate this divergence, the substitution we use to extract the temperature dependence of the expression renders this cutoff temperature dependent. We ignore this problem at this 
point, as we expect it to be an IR artifact that will be cured upon resummation of the appropriate diagrams.

The linear divergence arises as:

$$
-g^{2} T \int \frac{d^{3} p}{(2 \pi)^{3}} \frac{1}{2|p| 4 \pi|\theta p|}=-\frac{1}{4(2 \pi)^{2}} \frac{g^{2} T}{\theta} \Lambda .
$$

As discussed above, this divergence contributes to the entropy of the system, but not to the internal energy. We therefore attribute it to the degeneracy of the winding states as discussed in the text.

\section{B. IR divergences in $g^{2} \phi^{4}$}

The only potential infrared divergence in the mean field expression (4) for the free energy stems from the term

$$
\beta V\left\langle\int d^{4} x \phi(x) * \phi(x) * \phi(x) * \phi(x)\right\rangle .
$$

The non-planar contribution to this term is obtained from equation (2.1) by replacing the bare by the dressed propagators:

$$
-g^{2} T^{2} \sum_{n, l} \int \frac{d^{3} p}{(2 \pi)^{3}} \frac{d^{3} k}{(2 \pi)^{3}} \frac{e^{i p \theta k}}{\left(\frac{4 \pi^{2} n^{2}}{\beta^{2}}+p^{2}+M^{2}+g^{2}\left\langle\phi^{2}\right\rangle_{\theta p}\right)\left(\frac{4 \pi^{2} l^{2}}{\beta^{2}}+k^{2}+M^{2}+g^{2}\left\langle\phi^{2}\right\rangle_{\theta k}\right)},
$$

where $\left\langle\phi^{2}\right\rangle$ is given to leading order by equation (4.3). For small $p$ and small $k$, both terms in the denominator of the above expression blow up and no infrared divergence can occur. For small $p$ and large $k$ (or vice versa), we can ignore $\left\langle\phi^{2}\right\rangle_{\theta k}$ and perform the $d^{3} k$ integral as in (2.2). Upon performing the sums, we obtain for small $p$

$$
-g^{2} \int \frac{d^{3} p}{(2 \pi)^{3}} \frac{1+2 n_{\beta}(|p|)}{2 \sqrt{p^{2}+M^{2}+g^{2}\left\langle\phi^{2}\right\rangle_{\theta p}}} \frac{2}{8 \pi^{2}|\theta p|^{2}} .
$$

As one factor of $|\theta p|^{-1}$ is absorbed by the $\left\langle\phi^{2}\right\rangle_{\theta p}$ under the square root, this expression is finite in the infrared. 


\section{References}

[1] A. Connes, M. R. Douglas and A. Schwarz, "Noncommutative geometry and matrix theory: Compactification on tori," JHEP 9802, 003 (1998) [hep-th/9711162].

[2] D. Bigatti and L. Susskind, "Magnetic fields, branes and noncommutative geometry," [hep-th/9908056].

[3] N. Seiberg and E. Witten, "String theory and noncommutative geometry," JHEP 9909, 032 (1999) [hep-th/9908142].

[4] S. Minwalla, M. Van Raamsdonk and N. Seiberg, "Noncommutative perturbative dynamics," [hep-th/9912072].

[5] T. Filk, "Divergencies in a field theory on quantum space," Phys. Lett. B376, 53 (1996).

[6] M. Li, "Comments on supersymmetric Yang-Mills theory on a noncommutative torus," hep-th/9802052.

[7] N. Nekrasov and A. Schwarz, "Instantons on noncommutative R**4 and $(2,0)$ superconformal six dimensional theory," Commun. Math. Phys. 198, 689 (1998) [hep-th/9802068].

[8] M. Berkooz, "Non-local field theories and the non-commutative torus," Phys. Lett. B430, 237 (1998) [hep-th/9802069].

[9] J. C. Varilly and J. M. Gracia-Bondia, "On the ultraviolet behavior of quantum fields over noncommutative manifolds," Int. J. Mod. Phys. A14, 1305 (1999) [hep-th/9804001].

[10] M. Chaichian, A. Demichev and P. Presnajder, "Quantum field theory on noncommutative space-times and the persistence of ultraviolet divergences," hep-th/9812180.

[11] C. P. Martin and D. Sanchez-Ruiz, "The one-loop UV divergent structure of U(1) Yang-Mills theory on noncommutative R**4," Phys. Rev. Lett. 83, 476 (1999) [hepth/9903077].

[12] M. M. Sheikh-Jabbari, "Renormalizability of the supersymmetric Yang-Mills theories on the noncommutative torus," JHEP 9906, 015 (1999) [hep-th/9903107].

[13] T. Krajewski and R. Wulkenhaar, "Perturbative quantum gauge fields on the noncommutative torus," hep-th/9903187.

[14] S. Cho, R. Hinterding, J. Madore and H. Steinacker, "Finite field theory on noncommutative geometries," hep-th/9903239.

[15] E. Hawkins, "Noncommutative regularization for the practical man," hep-th/9908052. 
[16] N. Ishibashi, S. Iso, H. Kawai and Y. Kitazawa, "Wilson loops in noncommutative YangMills," hep-th/9910004.

[17] I. Chepelev and R. Roiban, "Renormalization of quantum field theories on noncommutative $\mathrm{R}^{* *}$ d. I: Scalars," hep-th/9911098.

[18] T. Harmark and N. A. Obers, "Phase structure of non-commutative field theories and spinning brane bound states," hep-th/9911169.

[19] C. Chu and F. Zamora, "Manifest supersymmetry in non-commutative geometry," hepth/9912153.

[20] S. Iso, H. Kawai and Y. Kitazawa, "Bi-local fields in noncommutative field theory," hep-th/0001027.

[21] Y. Imamura, "T-duality of non-commutative gauge theories," JHEP 0001, 039 (2000) [hep-th/0001105].

[22] S. Terashima, "On the equivalence between noncommutative and ordinary gauge theories," hep-th/0001111.

[23] R. Cai and N. Ohta, "On the thermodynamics of large N non-commutative super YangMills theory," hep-th/9910092.

[24] A. Hashimoto and N. Itzhaki, "On the hierarchy between non-commutative and ordinary supersymmetric Yang-Mills," JHEP 9912, 007 (1999) [hep-th/9911057].

[25] I. Y. Aref'eva, D. M. Belov and A. S. Koshelev, "Two-loop diagrams in noncommutative phi**4(4) theory," hep-th/9912075.

[26] M. Hayakawa, "Perturbative analysis on infrared aspects of noncommutative QED on $\mathrm{R}^{* *} 4$," hep-th/9912094.

[27] G. Arcioni and M. A. Vazquez-Mozo, "Thermal effects in perturbative noncommutative gauge theories," JHEP 0001, 028 (2000) [hep-th/9912140].

[28] H. Grosse, T. Krajewski and R. Wulkenhaar, "Renormalization of noncommutative Yang-Mills theories: A simple example," hep-th/0001182.

[29] J. J. Atick and E. Witten, "The Hagedorn Transition And The Number Of Degrees Of Freedom Of String Theory," Nucl. Phys. B310, 291 (1988). 